\title{
The growth of non-solid neoplastic lung nodules is associated with low PD L1 expression, irrespective of sampling technique
}

\author{
Chandra Bortolotto' , Claudio Maglia², Antonio Ciuffreda ${ }^{3}$, Manuela Coretti ${ }^{3}$, Roberta Catania', \\ Filippo Antonacci ${ }^{4}$, Sergio Carnevale ${ }^{5}$, Ivana Sarotto ${ }^{6}$, Roberto Dore ${ }^{1}$, Andrea Riccardo Filippi ${ }^{7}$, Gabriele Chiara ${ }^{2}$, \\ Daniele Regge ${ }^{2}$, Lorenzo Preda ${ }^{8}$, Patrizia Morbini ${ }^{5}$ and Giulia Maria Stella ${ }^{3^{*}}$ (1)
}

\begin{abstract}
Background: Few data are known regarding the molecular features and patterns of growth and presentation which characterize those lung neoplastic lesions presenting as non-solid nodules (NSN).

Methods: We retrospectively reviewed two different cohorts of NSNs detected by CT scan which, after transthoracic fine-needle aspiration (FNA) and core needle biopsy (CNB) received a final diagnosis of malignancy. All the enrolled patients were then addressed to surgical removal of lung cancer nodules or to exclusive radiotherapy. Exhaustive clinical and radiological features were available for each case.

Results: In all 62 analysed cases the diagnosis of adenocarcinoma (ADC) was reached. In cytologic samples, EGFR activating mutations were identified in 2 of the 28 cases (7\%); no case showed ALKJEML4 or ROS1 translocations. In the histologic samples EGFR activating mutation were found in 4 out of 25 cases (16\%). PD-L1 immunostains could be evaluated in 30 cytologic samples, while the remaining 7 did not reach the cellularity threshold for evaluation. TPS was $<1 \%$ in 26 cases, $>1 \%<50 \%$ in 3, and $>50 \%$ in 1 . All surgical samples showed TPS $<1 \%$. Of the 17 cases that could be evaluated on both samples, 15 were concordantly TPS 0 , and 2 showed TPS $>1 \%<50$ on the biopsy samples. TPS was $<1 \%$ in 14 cases, $>1 \% /<5 \%$ in 4 cases, $>5 \% /<50 \%$ in 2 cases, $>50 \%$ in 1 case.

Conclusions: Overall PD-L1 immunostaining documented the predominance of low/negative TPS, with high concordance in FNA and corresponding surgical samples. It can be hypothesized that lung ADC with NSN pattern and predominant in situ (i.e. lepidic) components represent the first steps in tumor progression, which have not yet triggered immune response, and/or have not accumulated a significant rate of mutations and neoantigen production, or that they belong to the infiltrated-excluded category of tumors. The negative prediction of response to immunomodulating therapy underlines the importance of rapid surgical treatment of these lesions. Notably, cell block cytology seems to fail in detecting EGFR mutations, thus suggesting that this kind of sampling technique should be not adequate in case of DNA direct sequencing.
\end{abstract}

Keywords: Imaging, Biopsy, Nodule, Lung cancer, PD-L1, Molecular profiling

\footnotetext{
*Correspondence: g.stella@smatteo.pv.it

${ }^{3}$ Department of Medical Sciences and Infective Diseases, Unit of Respiratory Diseases, IRCCS Policlinico San Matteo Foundation and University of Pavia Medical School, 27100 Pavia, Italy Full list of author information is available at the end of the article
}

\section{Background}

Non-solid nodules (NSNs) are commonly detected at thoracic CT scan. They are identified as circumscribed areas of increased lung attenuation with preservation of the bronchial and vascular margins and are also

(c) The Author(s) 2020. This article is licensed under a Creative Commons Attribution 4.0 International License, which permits use, sharing, adaptation, distribution and reproduction in any medium or format, as long as you give appropriate credit to the original author(s) and the source, provide a link to the Creative Commons licence, and indicate if changes were made. The images or other third party material in this article are included in the article's Creative Commons licence, unless indicated otherwise in a credit line to the material. If material is not included in the article's Creative Commons licence and your intended use is not permitted by statutory regulation or exceeds the permitted use, you will need to obtain permission directly from the copyright holder. To view a copy of this licence, visit http://creativeco mmons.org/licenses/by/4.0/. The Creative Commons Public Domain Dedication waiver (http://creativecommons.org/publicdomain/ zero/1.0/) applies to the data made available in this article, unless otherwise stated in a credit line to the data. 
referred to as a ground glass opacity [1]. A defined NSN can be either partly solid (part of the nodule completely obscures the underlying lung parenchyma) or pure nonsolid [2]. They can be a manifestation of inflammation, infection, or fibrosis, but they can also be precursors of lung cancer, mainly adenocarcinoma (atypical adenomatous hyperplasia). Notably, persistent lesions have a high likelihood of malignancy. The ELCAP study reported a malignancy rate of $34 \%$ for all NSNs, $18 \%$ for pure ground glass lesions and $63 \%$ for partly solid lesions. Overall the latter featured even higher malignancy rates reaching up to $75 \%$ in other reports [3]. CT-guided needle biopsy of ground glass lesions is a useful diagnostic approach with acceptable complication rates approaching those of solid lesions [4-7]. It has been reported that in mixed nodules the diagnostic accuracy is influenced by the ground glass component, being lower in pure non-solid opacities [5].

Personalized approach to lung cancer generally requires tissue samples in order to perform exhaustive screening of molecular defects in actionable target genes. We and others showed the feasibility of tumor mutational screening in aspirated lung cancer cells (fine needle aspiration, FNA) obtained through imaging guidance [8-10]. At the same time, many other data underlined reliability of core needle biopsy (CNB) for lung cancer molecular profiling, even if some reports documented a slightly higher rate of complications [11-13]. More recently, both FNA cytology and CNB have been reported to be accurate, feasible and safe procedures for PD-L1 testing both retrospectively and in prospective studies [14-16]. It should be underlined that few data are known on the molecular features which characterize neoplastic lesions presenting as NSN, and poor information are available regarding the specific pattern of tumour presentation and growth as well as the diagnostic accuracy of different bioptic approaches in these settings. To explore these issues, the present study is focused on the analysis of the radiological and molecular aspects of two series of nonsolid neoplastic nodules obtained through two different transthoracic approaches and aimed at generating their matched radio-pathologic profiles. Moreover, we could compare the results obtained on cytological specimens with the subsequent corresponding resected surgical samples to validate the bioptic preliminary data (Fig. 1).

\section{Patients and methods}

\section{Patients identification and selection}

We retrospectively reviewed the $\mathrm{CT}$ scans of patients who were followed at IRCCS San Matteo Hospital Foundation and underwent transthoracic CT-guided fine needle aspiration of suspected pulmonary lesions from October 2016 to December 2017 to select the cases showing imaging features consistent with non-solid and mixed lung nodules. We identified 40 cases of which 37 subsequently received the diagnosis of malignancy, whereas for the remaining three cases the diagnosis was coherent with epithelioid aggregates (1 case), granulomas (1 case), sarcoidosis (1 case). Based on early disease stage, all the enrolled patients were then addressed to surgical removal of lung cancer nodules and for 20 out of the 37 analysed cases, the post-operative histology was available for analysis. To further validate this work, we also retrospectively reviewed the CT scans of patients followed at IRCCS Candiolo Cancer Institute, who underwent transthoracic CT-guided core needle biopsy of suspected pulmonary lesions from March 2016 to June 2018. During this temporal range, we identified 25 cases of NSNs that subsequently received the diagnosis of lung cancer.
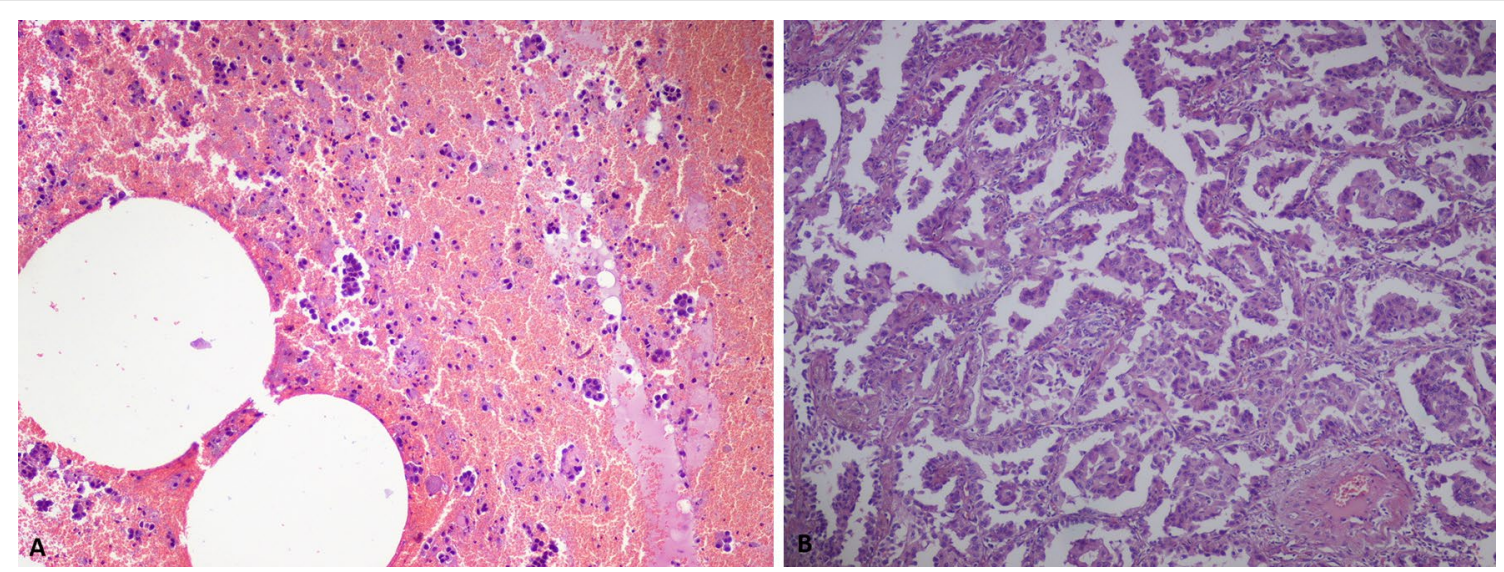

Fig. 1 Morphology of non-solid nodules samples obtained through CT-guided biopsy. a Cell-block of CT-guided biopsy with neoplastic epithelial cell with secretive features, arranged in strains and micro-papillae (H\&E, 100x). b Adenocarcinoma of the lung with prevalent lepidic growth. Invasive features are focally present in the neoplasia (H\&E, 100x) 
As above indicated, based on the different stages of presentation, the enrolled patients were then addressed to surgical removal of lung cancer nodules or to exclusive radiotherapy.

For each case exhaustive clinical, histological, molecular and procedural data were collected and available in Tables 1 and 2.

\section{CT-guided biopsy procedure}

CT-guided biopsies were performed using different multidetector scanners. The biopsies were performed both with conventional and fluoroscopic approach, depending on the nodule characteristics and location. During a fluoroscopic biopsy the operator stayed close to the patients inside the gantry room; the table could be moved using a joystick and the image can be created in real time using a pedal. Images can be obtained with a frame rate up to 3 frames/s. Generally, only in the first step of the procedure a real fluoroscopic vision was needed in order to study the movement of the nodule, the ribs and the diaphragm (for basal nodules); in the latter stages only one-shot images were needed to check the position of the needle compared to the nodule. The gantry could be tilted up to + or $-22^{\circ}$ to avoid ribs or vessels. Conventional CT approach is characterized by short spiral acquisition to check the position of the needle, performed without the presence of the operator inside the gantry room. Spiral acquisition allowed progressive adjustments of the needle position, performed in-bore, between different spiral acquisitions.

Dose-length product of a biopsy procedure was generally around $100 \mathrm{mGy}^{*} \mathrm{~cm}$ (DLP) ranging from 50 to $500 \mathrm{mGy}^{*} \mathrm{~cm}$ on the patients. The dose to the operators varied on the same range and never exceeded the regulatory limits for eye-lens, hands and body even for operators that performed up to 200 procedure a year (generally no less than 4 procedure a week). Among the personal protective equipment for fluoroscopic procedure, there were lead apron, glasses and gloves.

CT-guided biopsies were performed both as fineneedle aspiration biopsy (FNAB) and core biopsy (CB). FNAB was performed with chiba needles (HS Diva, HS, Italy) ranging from 24G to $20 \mathrm{G}$ (several needles had a $24 \mathrm{G}$ tip and $22 \mathrm{G}$ body in order to be less invasive on the pleura but at the same time offering a wider diameter to avoid clogs). The length of the needle could be 10 or $15 \mathrm{~cm}$. The aspiration and the to-and-fro movement ensured the capillary draining of the material inside a small syringe connected to the needle. No automatic aspiration pistols were used since the space inside the gantry is limited especially on the lateral sides of the patient; we avoided automatic aspiration especially for non-solid nodules since a close control of the aspiration strength avoid exceeding in aspiration which results in an alveolar haemorrhage. When the tip of the needle was inside the lesion the aspiration time could be modulated by the operator on the basis of the quantity and quality (perceived by the operator) of the material aspirated. The tip of the needle was small enough to be directed and inserted on the solid portion of mixed nodules in order to sample the locations more likely to release good quality material with a higher percentage of neoplastic cells. Since in non-solid nodules only a small amount of cells plastered the alveoli surface one of the advantages of the FNAB was to reach different portion of the nodules in order to "enrich" the material by sampling different areas of the nodule. Generally, we tried at least 3 different location; but it is hypothetically possible to reach every portion of the nodule with the same needle insertion. Core-biopsy needle were semi-automatic models ranging from 20G to 16G (BiopsyBell, BPB Medical, Italy and Precisa, HS, Italy), which can retrieve samples of 10 or $20 \mathrm{~mm}$ in length. The approach was similar to that of FNA biopsy, but the retrieval of the micro-histologic sample is performed with a two-step procedure: advancement of the needle inside the lesion and firing of the mechanism.

Complication rates were extremely low (around 25\% of any kind and grade of complication; less than $7 \%$ of clinically relevant complications and 3\% of complications requiring hospitalization). Material obtained from FNA was fixed in formalin in order to obtain a cell/cyto-block for the pathologist; material obtained from CBN was also fixed in formalin after minimal manipulation to remove it from the needle cradle.

All patients who were routinely assuming warfarin or platelet antiaggregants were asked to stop their therapies and to shift to low molecular weight heparin that was then suppressed the day before the procedure. Platelets counts at baseline were within normal range for all patients.

\section{Cytological, histological and molecular techniques}

A rapid on-site evaluation (ROSE) cytopathology service was not available. In case of FNA, no aspirative smear was assessed, and all the obtained material was immediately processed with $10 \%$ formalin fixation and paraffin embedding to obtain a cell block. It is widely accepted that cell block technique increases diagnostic accuracy with efficient results [17-19]. For both cell block and histologic specimens, haematoxylin and eosin (H\&E)-stained sections were evaluated by a pulmonary cytopathologist; immunohistochemical stains were performed whenever required for the precise characterization of neoplastic cells [20, 21]. According to recent guidelines [22], non-small cell lung cancer 


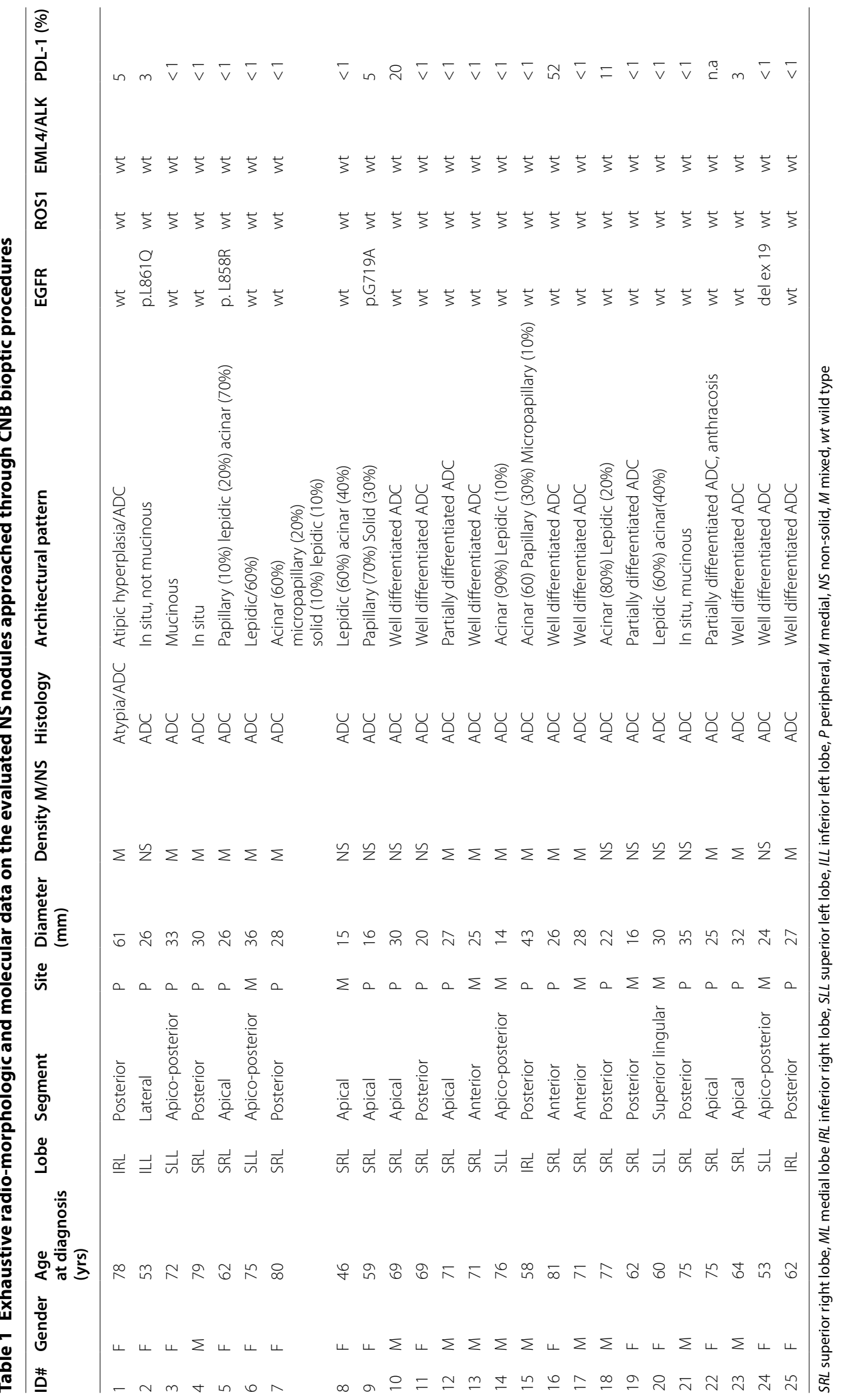




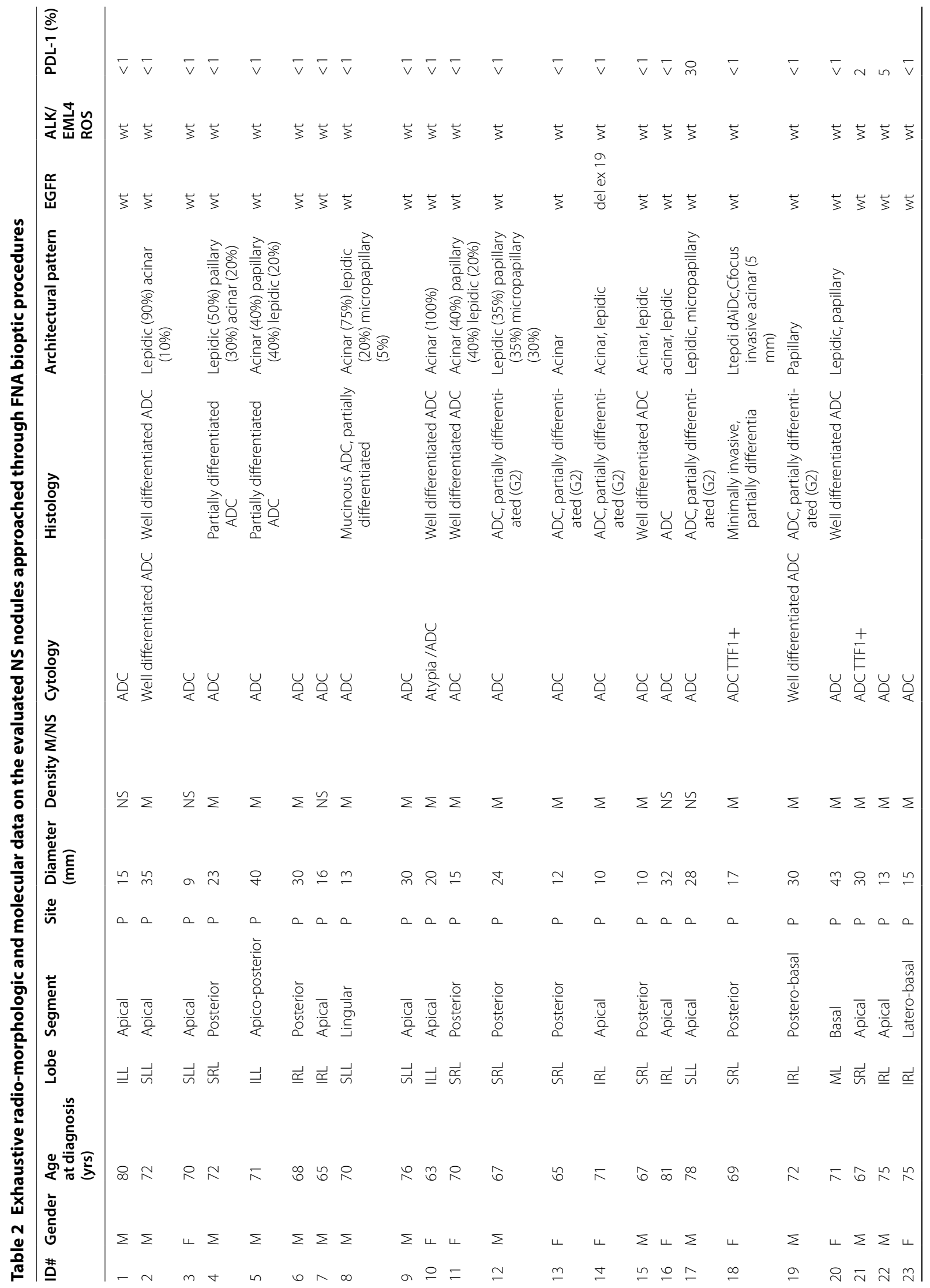




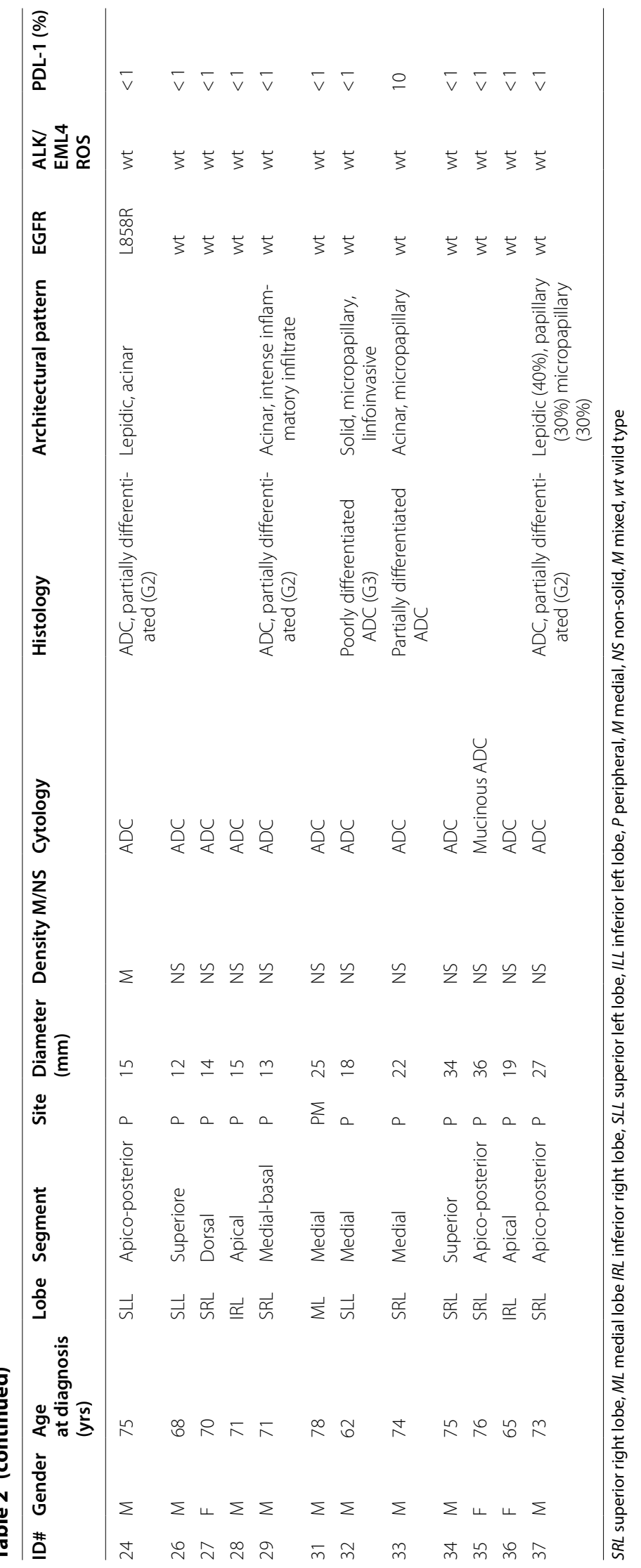


(NSCLC) cytological samples were submitted to immunohistochemical assay of PD-L1 expression levels using a laboratory-developed test designed to optimize the use of anti-PD-L1 22C3 antibody (Dako) on the Omnis autostainer (Dako) with Envision FLEX (Dako) revelation system. Tumor proportion score (TPS) was evaluated according to published guidelines [23]. Lung resection specimens were fixed in formalin and embedded in paraffin for routine histopathological evaluation; histopathological patterns of ADC were assed and quantified with $5 \%$ increments according to most recent guidelines (archives 2011). In cases diagnosed as primary lung adenocarcinomas (ADC) mutational analysis of the coding sequence of EGFR-TK domain and evaluation of $A L K / E M L 4$ and ROS1 translocations were performed as already published [24-26] on both cytologic and surgical specimens when required by the oncologist.

\section{Statistical analysis}

Descriptive statistics has been obtained for all variables assessed in the study population. Groups have been compared by means of parametric or nonparametric tests for quantitative variables and Pearson's Chi-square for categorical variables.

\section{Results}

\section{Clinical and radiological features of NSNs}

We identified 62 patients who were diagnosed at our Institutions with lung malignant NSNs. Of them, 26 were females $(41.9 \%)$ and $36(58.1 \%)$ males; the mean age at diagnosis was 69,35 years. Thirty-four patients were never smokers, while 14 were past smokers and only 4 was a current smoker. Smoking history was unknown in 9 cases. Twenty-five (40.3\%) out of the 62 evaluated patients referred a previous diagnosis of cancer and were in remission at time of enrolment; one patient was assuming immunosuppressive drugs due to previous heart transplantation. Forty out of the 62 lesions were in the right lung, 30 in the upper lobe, 8 in the lower and 2 in the middle one, respectively. Seventeen were in the left lung, 10 in the upper lobe, 6 in the lower and 1 in the lingula, respectively. Overall, in 26 cases consisted of basal parenchymal lesion lesions, more difficult to be approached. Based on nodule localization, the procedural approach was posterior or anterior. Most lesions occurred peripherally within the lung parenchyma (48 patients, $77.4 \%$ ); while 13 cases featured peri-mediastinal nodules. Twenty-nine (46.77\%) of the analysed nodules displayed a contact with the pleural layer. Of them, 4 nodules grew in contact with the fissure, in 16 a small peduncle was detected whereas the remaining 9 featured a wide interface with the visceral pleura. The vast majority of nodules displayed a round/ovalar shape with irregular margins. The aerated lung run of the needle was between $1 \mathrm{~mm}$ and $60 \mathrm{~mm}$ respectively, the minimal distance from middle-large veins and arteries (segmental, lobar and main pulmonary) was of $3 \mathrm{~mm}$. In 37 (59.6\%) cases, nodules had mixed or semi-solid appearance, the remaining were pure non-solid. All but seven lesions were small, displaying a diameter $\leq 3 \mathrm{~cm}$; in only two case the diameter was of $5 \mathrm{~cm}$ and $6 \mathrm{~cm}$, respectively.

Thirty-seven out of all the patients underwent transthoracic FNA TC-guided biopsy (needle: 24 gauge) leading to a diagnosis of NSCLC (ADC) in 31; in six cases, the diagnosis was inconclusive (suspicious for neoplastic cells); in 3 cases out of the latter a second FNA was performed, leading to a conclusive ADC diagnosis. In 25 patients transthoracic TC-guided CNB (needle: 18 gauge) was performed and allowed the diagnosis of NSCLC (ADC) in all cases.

The diagnosis of lung ADC was reached in all cases on the sole basis of cell morphology and architecture on $\mathrm{H} \& \mathrm{E}$ stained slides derived from both FNA and CNB tissue samples; immunohistochemical staining was required in 3 cases to exclude metastasis form a previous extrapulmonary ADC.

Overall fourteen out of the 62 cases early minor complications (22.5\%). In detail, among the 37 nodules biopsied through FNA, minor complications aroused in 6 cases $(9.6 \%)$, one patient presented mild haemoptysis rapidly solved after tranexamic acid infusion; whereas in the remaining five a small pneumothorax could be demonstrated after the procedure. In the cohort of the 25 nodules reached through by CNB, 8 (12.8\%) cases presented early small pneumothorax demonstrated after the procedure. Major complications regarded the occurrence of complete pneumothorax which was managed by chest tube insertion and drainage in one case and in two case in the cohort of FNA and CNB, respectively. No late complications or pleural or bleeding/haemothorax occurred in the cohort of FNA procedures whereas in two cases who underwent $\mathrm{CNB}$ a minimum haemothorax occurred, without any further therapeutic management required. No late complications occurred. All the 62 patients did not refer significant pain during and after procedure (Numerical Rating Scale NRS $\leq 3$ ).

\section{Morphologic and molecular profiling of NSNs}

Based on early disease stage, all the enrolled patients were addressed to surgical resection of lung cancer nodules to exclusive radiotherapy or to exclusive radiotherapy.

In 20 out of the 37 cases diagnosed through FNA, surgical resection was conducted at our Institution. The latter allowed the unique opportunity to perform a complete morphological and molecular match between the FNA-derived samples and their corresponding removed 
tissue. Surgical resection confirmed the cytological diagnosis of invasive or minimally invasive ADC in all the 20 cases. One case was in situ, 4 were minimally invasive and 15 invasive. The predominant histopathological pattern was lepidic in 7 (35\%) cases, papillary in $6(30 \%)$, acinar in 3 (15\%), and solid in 1 (5\%); 3 cases (15\%) had an equivalent proportion of acinar and papillary, acinar and lepidic, and papillary and lepidic components. Seventeen cases showed the coexistence of more patterns: 4 acinar and lepidic, 1 each lepidic and papillary, papillary and acinar, solid and micropapillary, 3 lepidic acinar and papillary, 2 acinar papillary and micropapillary, two lepidic papillary and micropapillary, 2 lepidic, acinar, papillary and micropapillary. Three cases showed a homogeneous pattern, 1 acinar, 1 papillary and 1 lepidic. Overall, a lepidic component was present in 13 cases, acinar in 14 , papillary in 12 , micropapillary in 8 , and solid in 1 . No specific pattern could be recognized in FNA cytological samples but unequivocal papillae in the case with exclusive papillary architecture. The occurrence of unsatisfactory FNA samples was not associated with any specific histological pattern. No acinar components in non-solid nodules as acinar components could account for solid portion of the mixed subsolid-nodules.

PD-L1 immunostains could be evaluated in 30 cytological samples, while the remaining 7 did not reach the cellularity threshold for evaluation. TPS was $<1 \%$ in 26 cases, $>1 \% /<50 \%$ in 3 , and $>50 \%$ in 1 . All surgical samples showed TPS $<1 \%$. Of the 20 cases that could be evaluated on both cytologic and surgical samples, 18 were concordantly TPS 0 , and 2 showed TPS $>1 \%<50$ on the biopsy samples.

EGFR mutations were identified in 2 of the 28 cases (7\%) with adequate material (one exon 19 deletion and L858R); no case showed ALK/EML4 or ROS1 translocations.

Eight of the 25 cases diagnosed through CNB were patients that after the biopsy decided to continue the diagnostic-therapeutic path in other structures. In eleven of the 25 cases, surgical resection was performed. Surgical resection confirmed the cytological diagnosis of invasive or minimally invasive ADC in all 11 cases. The predominant histopathological pattern was acinar in 8 cases, papillary in 2 and acinar in 1.

PD-L1 expression could be evaluated in 22 samples, while the remaining three samples did not reach the cellularity threshold for evaluation. TPS was $<1 \%$ in 15 cases, $>1 \% /<5 \%$ in 4 cases, $>5 \% /<50 \%$ in 2 cases, $>50 \%$ in 1 case.

EGFR mutations were identified in 4 of the 25 cases (16\%) with adequate material (one exon 19 deletion, L858R, L861Q, G719A); no case showed $A L K / E M L 4$ or ROS1 translocations.
Overall, being the population evaluated in the study homogenous, the Chi squared analysis (with degree of statistical freedom $\mathrm{k}=1$ ) of two distribution regarding the EGFR mutational profiling, allows us to reject the null hypothesis that the two groups FNA biopsy and CNB biopsy) are the same, whereas regarding the PD-L1 expression the two frequencies are coherent with the expected one. In other words, by comparing the two bioptic techniques, FNA and cell block cytology result in an EGFR mutational frequency lower than the expected one. In conclusion, cell-block cytology appears to be adequate for IHC stain but not in case of DNA direct sequencing.

\section{Discussion}

Despite relevant progresses in personalized approach to lung cancer, many aspects remain to be clarified. One of the most important issue is that lung cancers have different pathologic features. In this perspective non-solid malignant lung nodules define a still obscure neoplastic entity. Although many reports are already available regarding diagnostic approach to NSNs, fewer data exist in terms of their molecular and pathological characterization. We retrospectively reviewed a relevant cohort of NSN that were first confirmed as malignant on CTguided FNA and were subsequently resected. Importantly, we further analysed a corresponding cohort of NSNs which were approached by core biopsy. This gave us the unique opportunity to perform exhaustive pathological and molecular profiling of tumor material on both cyto- and histological specimens focusing on two complementary goals: (i) the characterization of the histopathological and molecular profiles of non-solid neoplastic growth; (ii) the validation of a panel of molecular markers on cytological samples as consistent (predictive) to those of the whole lesion.

The findings of our study indicate that CT-guided FNA is a safe, feasible and accurate procedure as CB for the diagnosis of NSNs and mixed neoplastic nodules. In $83 \%$ of cases, a conclusive diagnosis was reached on the first sampling, while the remaining were classified as suspicious, which in 2 cases lead to surgical resection with intraoperative confirmation of neoplastic nature, and in 3 to a second CT-FNA with conclusive diagnosis. As expected, and coherently with the most published data, all malignant NSNs where diagnosed as ADC. By comparing data obtained on the two cohorts, several aspects deserve to be underlined. The first is that the development of the cell block method, a complementary approach to conventional FNA cytology, is responsible for the $100 \%$ accuracy of tumor histotype definition on FNA cytology. The possibility to submit cell block sections to IHC staining also allows a conclusive differential 
diagnosis in patients with a previous history of extrapulmonary ADC. However, the most relevant advantage associated with cell block is the possibility of using these samples for molecular subtyping and definition of immunotherapy response predictive markers. Reflex PD-L1 ICH stain is recommended, considering that 4 of the 7 (19\% of the total) FNA samples with insufficient cellularity were from cell block recuts obtained after molecular analysis.

As far as histomorphology is concerned, most cases showed mixed patterns as generally reported; acinar, lepidic and papillary architectures were almost equally common in different combinations, but lepidic pattern were more frequently predominant. No specific architectural pattern could be recognised on FNA. PD-L1 immunostains documented the predominance of low/negative TPS, with high concordance between FNA and CB and corresponding surgical samples. High TPS was observed in only 1 FNA sample and 1 CB sample, both not submitted to surgery. Low PD-L1 expression rates have previously been documented in in situ and minimally invasive ADC (0\%), and in ADC with lepidic (0\%) and acinar (36\%) components, while papillary ADC scores are intermediate [27-29]. Similarly, studies aimed at correlating PD-L1 expression with CT scan features documented that PD-L1 was negatively associated with the presence of ground glass opacities surrounding the tumor nodule [30, 31]. No explanations have been provided so far for this phenomenon; it can be hypothesized that lung ADC with NSN pattern and predominant in situ (i.e. lepidic) components represent the first steps in tumor progression, which have not yet triggered adaptive immune-resistance through $\mathrm{T}$ cell activation and ifn-gamma secretion, and/ or have not accumulated a significant rate of mutations and neoantigen production, or that they belong to the infiltrated-excluded category of tumors [32].The negative prediction of response to immunomodulating therapy underlines the importance of rapid surgical treatment of these lesions.

The vast majority of patients did not refer a smoking history. Despite that, a very low rate of actionable mutations was identified in our cohort: no alterations were detected in $A L K$ and ROS1 genes, EGFR mutational frequency was lower than expected in this population ( $7 \%$ vs $\approx 15 \%$ of the expected one) in the FNA cohort, whether by sequencing $\mathrm{CBN}$-derived samples the mutational frequency is coherent to the expected (16\%). While the first result is consistent with literature data reporting a low rate of ALK rearrangement in papillary or lepidic predominant, in situ and minimally invasive ADC [33, 34], most published studies obtained in Asian populations agree on the high prevalence of EGFR mutations in these ADC subgroups [32, 33], and more generally in nodules with ground-glass CT features [35]. Within the limit of the cohorts analysed, these data suggest different tracks leading to the development of solid and non-solid tumors.

\section{Conclusions}

To the best of our knowledge, this is the first report which aims at comparing-in non solid adenocarcinomas-two CT-guided diagnostic approaches. Interestingly, they result comparable regarding $\mathrm{ICH}$ staining, as demonstrated by PD-L1 expression analysis that is coherently low in matched cytologic, histologic and surgical samples. Notably, in case of DNA direct sequencing, FNA cell-block specimens seem to be not fully performing mainly due to the low DNA mass. It is conceivable that DNA extracted from cell blocks should be more efficiently processed through next generation sequencing in high-throughput laboratories, but more data are required to validate this suggestion. Overall, at least in case of NSNs, the diagnostic approach should be decided mainly by taking under consideration the further process of the obtained sample.

\section{Abbreviations}

ADC: Adenocarcinoma; FNA: Fine needle aspiration; CNB: Core needle biopsy; CT: Computed tomography; NSN: Non solid nodule; DLP: Dose-length product; PD-L1: programmed death ligand 1; TK: Tyorisine kinase; TPS: Tumor proportion score.

\section{Acknowledgements \\ The authors thanks E. Pozzi for fruitful discussion and A. Marchelli for careful revision of the manuscript.}

\section{Authors' contributions}

GMS, FA, CB, PM, CM, DR and RD designed the study and analysed data, AC, MC, MCM, RC, CM and SC collected data, GMS, CB and PM wrote the paper. In memory of Francesco Valentino, Department of Medical Sciences and Infective Diseases, Unit of Oncology, IRCCS Policlinico San Matteo Foundation and University of Pavia Medical School, Pavia, Italy. All authors read and approved the final manuscript.

\section{Funding}

Ricerca Corrente-IRCCS Fondazione Policlinico San Matteo to GMS. GMS takes responsibility for the content of the manuscript, including the data and analysis.

\section{Availability of data and materials}

The datasets used and/or analysed during the current study are available from the corresponding author on reasonable request.

\section{Ethics approval and consent to participate}

The study has been approved by Ethic Committee as a part of the Ricerca Corrente project entitled: "Trattamento del Non Small Cell Lung Cancer (NSCLC) in stadio avanzato modulato sul profilo farmaco-genomico individuale".

\section{Consent for publication}

Patients evaluated in the study were enrolled in the above-mentioned protocol and signed informed consent.

\section{Competing interests}

$\mathrm{CB}$ is consultant for Bracco Imaging Italia and Doc. Congress. The authors declare that they have no competing interests. 


\begin{abstract}
Author details
${ }^{1}$ Department of Intensive Medicine, Unit of Radiology, IRCCS Policlinico San Matteo Foundation and University of Pavia Medical School, Pavia, Italy. ${ }^{2}$ Radiology Unit, IRCCS Candiolo Cancer Institute and University of Turin Medical School, Candiolo, TO, Italy. ${ }^{3}$ Department of Medical Sciences and Infective Diseases, Unit of Respiratory Diseases, IRCCS Policlinico San Matteo Foundation and University of Pavia Medical School, 27100 Pavia, Italy. ${ }^{4}$ Department of Intensive Medicine, Unit of Cardiothoracic Surgery, IRCCS Policlinico San Matteo Foundation and University of Pavia Medical School, Pavia, Italy. ${ }^{5}$ Department of Molecular Medicine, Unit of Pathology, IRCCS Policlinico San Matteo Foundation and University of Pavia Medical School, Pavia, Italy. ${ }^{6}$ Unit of Pathology, IRCCS Candiolo Cancer Institute, Candiolo, TO, Italy. ${ }^{7}$ Department of Medical Sciences and Infective Diseases, Unit of Radiation Therapy, IRCCS Policlinico San Matteo Foundation and University of Pavia Medical School, Pavia, Italy. ${ }^{8}$ Department of Clinical-Surgical, Diagnostic and Pediatric Sciences, University of Pavia, Pavia, Italy.
\end{abstract}

Received: 8 January 2020 Accepted: 26 January 2020 Published online: 03 February 2020

\section{References}

1. Hansell DM, Bankier AA, MacMahon H, McLoud TC, Müller NL, Remy J. Fleischner society: glossary of terms for thoracic imaging. Radiology. 2008;246(3):697-722.

2. Henschke Cl, Yankelevitz DF, Mirtcheva R, McGuinness G, McCauley D, Miettinen OS. ELCAP Group CT screening for lung cancer: frequency and significance of part-solid and nonsolid nodules. Am J Roentgenol. 2002;178(5):1053-7

3. Welch HG, Woloshin S, Schwartz LM, et al. Overstating the evidence for lung cancer screening: the international early lung cancer action program (I-ELCAP) study. Arch Intern Med. 2007;167:2289-95.

4. Hur J, Lee HJ, Nam JE, et al. Diagnostic accuracy of CT fluoroscopy-guided needle aspiration biopsy of ground-glass opacity pulmonary lesions. Am J Roentgenol. 2009;192:629-34.

5. Kim TJ, Lee JH, Lee CT, Jheon SH, Sung SW, Chung JH, Lee KW. Diagnostic accuracy of CT-guided core biopsy of ground-glass opacity pulmonary lesions. Am J Roentgenol. 2008;190(1):234-9.

6. Inoue D, Gobara H, Hiraki T, et al. CT fluoroscopy-guided cutting needle biopsy of focal pure ground-glass opacity lung lesions: diagnostic yield in 83 lesions. Eur J Radiol. 2012;81(2):354-9.

7. Hiraki T, Mimura H, Gobara H, et al. CT fluoroscopy-guided biopsy of 1,000 pulmonary lesions performed with 20-gauge coaxial cutting needles. Chest. 2009;136:1612-7.

8. Stella G, Scabini R, Inghilleri S, et al. EGFR and KRAS mutational profiling in fresh non-small cell lung cancer (NSCLC) cells. J Cancer Res Clin Oncol. 2013;139(8):1327-35.

9. Schuurbiers OC, Looijen-Salamon MG, Ligtenberg MJ, et al. A brief retrospective report on the feasibility of epidermal growth factor receptor and KRAS mutation analysis in transoesophageal ultrasound- and endobronchial ultrasound- guided fine needle cytological aspirates. J Thorac Oncol. 2010;5(10):1664-7.

10. Ulivi P, Romagnoli M, Chiadini E, et al. Assessment of EGFR and K-ras mutations in fixed and fresh specimens from transoesophageal ultrasound-guided fine needle aspiration in non-small cell lung cancer patients. Int J Oncol. 2012:41(1):147-52.

11. Chang YY, Chen CK, Yeh YC, Wu MH. Diagnostic feasibility and safety of CT-guided core biopsy for lung nodules less than or equal to $8 \mathrm{~mm}$ : a single-institution experience. Eur J Radiol. 2018;28(2):796-806.

12. Heerink WJ, de Bock GH, de Jonge GJ, Groen HJ, Vliegenthart R, Oudkerk M. Complication rates of CT-guided transthoracic lung biopsy: metaanalysis. Eur Radiol. 2017;27(1):138-48.

13. Sangha BS, Hague CJ, Jessup J, O'Connor R, Mayo JR. Transthoracic computed tomography-guided lung nodule biopsy: comparison of core needle and fine needle aspiration techniques. Can Assoc Radiol J. 2016;67(3):284-9.

14. Noll B, Wang WL, Gong Y, et al. Programmed death ligand 1 testing in non-small cell lung carcinoma cytology cell block and aspirate smear preparations. Cancer Cytopathol. 2018;126(5):342-52.
15. Heynmann JJ, Bulman WA, Swinarski D, et al. PD-L1 expression in nonsmall cell lung carcinoma: comparison among cytology, small biopsy, and surgical resection specimens. Cancer Cytopathol. 2017;125(12):896-907.

16. Tsai EB, Pomykala K, Ruchalski K, et al. Feasibility and safety of intrathoracic biopsy and reapet biopsy for evaluation of programmed cell death ligand-1 expression for immunotherapy in non-small cell lung cancer. Radiology. 2018;287(1):326-32.

17. Saqi A. The state of cell blocks and ancillary testing. Arch Pathol Lab Med. 2016:140(12):1318-22.

18. Khan S, Omar T, Michelow P. Effectiveness of the cell block technique in diagnostic cytopathology. J Cytol. 2012;29(3):177.

19. Balassanian R, Wool GD, Ono JC, et al. A superior method for cell block preparation for fine needle aspiration biopsies. Cancer Cytopathol. 2016:124:508-18.

20. Pelosi G, Fraggetta F, Pasini F, et al. Immunoreactivity for thyroid transcription factor-in stage 1 non-small-cell carcinomas of the lung. Am J Surg Pathol. 2001;25(3):363-72.

21. Pelosi G, Fabbri A, Bianchi F, et al. $\triangle N p 63(p 40)$ and thyroid transcription factor-1 immunoreactivity on small biopsies or cell blocks for typing nonsmall cell lung cancer: a novel two-hit sparing material approach. J Thor Oncol. 2012;7(2):281-90.

22. Kerr KM, Bubendorf L, Edelman MJ, et al. Second ESMO consensus conference on lung cancer: pathology and molecular biomarkers for non-smallcell-lung cancer. Ann Oncol. 2014;25:1681-90.

23. Ettinger DS, Wood DE, Akerley W, et al. NCCN Guidelines Insights: nonsmall cell lung cancer. J Natl Compr Canc Net. 2016;14(3):255-64.

24. Stella GM, Benvenuti S, Gramaglia D et al. MET mutations in cancers of unknown primary origin (CUPS). Hu Mut 2011; 32 (1).

25. Stella GM, Benvenuti S, Gramaglia D, et al. MET mutations in cancers of unknown primary origin (CUPs). Hu Mut. 2011;32(1):44-50.

26. Luk P, Selinger Cl, Mahar A, Cooper WA. Biomarkers for ALK and ROS in lung cancer: immunohistochemistry and fluorescent in situ hybridization. Arch Pathol Lab Med. 2016;140:922-8.

27. Ng Kee Kwong F, Laggner U, McKinney O, Croud J, Rice A, Nicholson AG. Expression of PD-L1 correlates with pleomorphic morphology and histological patterns of non-small-cell lung carcinomas. Histopathology. 2018;72(6):1024-32

28. Miyazawa T, Marushima H, Saji H, Kojima K, Hoshikawa M, Takagi M, Nakamura H. PD-L1 expression in non-small-cell lung cancer including various adenocarcinoma subtypes. Ann Thorac Cardiovasc Surg. 2019;25(1):1-9.

29. Yu R, He Z, Lou Y, Jiang H, Wu Y, Liu Z, Pan H, Han W. Clinical characteristics and programmed cell death ligand-1 expression in adenocarcinoma in situ and minimally invasive adenocarcinoma of lung. Oncotarget. 2017;8(58):97801.

30. Toyokawa G, Takada K, Okamoto T, et al. Computed tomography features of lung adenocarcinomas with programmed death ligand 1 expression. Clin Lung Cancer. 2017;18(6):e375-83.

31. Wu T, Zhou F, Soodeen-Lalloo AK, et al. The association between imaging features of TSCT and the expression of PD-L1 in patients with surgical resection of lung adenocarcinoma. Clin Lung Cancer. 2019;20(2):e195-207.

32. Binnewies M, Roberts EW, Kersten $\mathrm{K}$, et al. Understanding the tumo immune microenvironment (TIME) for effective therapy. Nat Med. 2018;24(5):541-50.

33. Tsuta K, Kawago M, Inoue E, et al. The utility of the proposed IASLC/ATS/ ERS lung adenocarcinoma subtypes for disease prognosis and correlation of driver gene alterations. Lung Cancer. 2013;81(3):371-6.

34. Hu H, Pan Y, Li Y, et al. Oncogenic mutations are associated with histological subtypes but do not have an independent prognostic value in lung adenocarcinoma. Onco Targets Ther. 2014;13(7):1423.

35. Kobayashi Y, Mitsudomi T, Sakao Y, Yatabe Y. Genetic features of pulmonary adenocarcinoma presenting with ground-glass nodules: the differences between nodules with and without growth. Ann Oncol. 2015:26:156-61.

\section{Publisher's Note}

Springer Nature remains neutral with regard to jurisdictional claims in published maps and institutional affiliations. 